SELECTED PAPER AT NCSP'19

\title{
Genetic-Algorithm-Based Evacuation Sign Arrangement Method Using Multi-Agent Simulation
}

\author{
Yuki Fujita and Hiroaki Kurokawa \\ School of Engineering, Tokyo University of Technology \\ 1404-1, Katakura-machi, Hachioji, Tokyo 192-0982, Japan \\ E-mail: hkuro@stf.teu.ac.jp
}

\begin{abstract}
In this study, for the case of evacuation in a disaster situation, we propose a method for automatically selecting installation spots for evacuation signs on a map of the local area. The proposed method is a genetic-algorithm-based method. In our method, the individuals represent the evacuation sign arrangement on the local map, and the objective function to be optimized is defined by the evacuation completion time and the number of evacuation signs. The evacuation completion time is calculated by multi-agent simulation, which is a method that can simulate social phenomena by defining simple agent rules.
\end{abstract}

\section{Introduction}

Powerful earthquakes have occurred around the world. Especially in Japan, many earthquake disasters have occurred. To reduce the damage, countermeasures against earthquakes such as seismic reinforcement are, of course, necessary. On the other hand, it is also important to evacuate quickly after an earthquake to prevent secondary disasters. It is possible to prompt efficient evacuation by the appropriate arrangement of evacuation signs.

Conventionally, for the arrangement of evacuation signs in a local area, expert knowledge of selection and experience in the local area are necessary for the selection of installation spots, and ineffective evacuation signs may be included in the arrangement depending on the ability of the person planning the evacuation sign arrangement. Therefore, a method of obtaining the optimal arrangement of evacuation signs is strongly required. A method of automatically selecting the installation spots of evacuation signs on a map of the local area provides a high-quality evacuation sign arrangement and has a positive influence on the formulation of regional disaster management plans.

In this study, we propose a method of optimizing the evacuation sign arrangement using multi-agent simulation. The proposed method is a genetic-algorithm-based method. The objective function consists of the evacuation completion time and the number of evacuation signs. The result of disaster evacuation obtained by multi-agent simulation is used to evaluate the individuals in the genetic algorithm.

\section{Evacuation Simulation by Multi-Agent Simulation}

Multi-agent simulation is a simulation framework using an agent that can recognize surrounding situations and act under arbitrary rules. By using multi-agent simulation with simple agent rules, it is possible to simulate various social phenomena. The evacuation simulation is one of the effective applications of multi-agent simulation, and the validity of the simulation has been shown in many conventional studies [1][2].

In this study, we carry out a simulation to evaluate the evacuation sign arrangement in a local area. For that purpose, we perform evacuation simulation taking into consideration local area factors[1]. In this conventional study, wide-area disaster evacuation simulation for arbitrary areas is made possible by considering the characteristics of evacuees and evacuation environments, which differ from region to region.

In the evacuation simulation in this study, an area of 600 $m$ squares is assumed. As shown in Fig. 1, a map of the area is divided into $20 \mathrm{~m}$ squares, and the simulation is performed on a field of $30 \times 30$ cells. Each cell contains the following information: the address, the coordinates of the road, the address of the neighboring cell, the size of the road, the maximum number of evacuees that can exist in the cell, the number of current evacuees in the cell, whether it is an intersection, whether an evacuation site is adjacent, and whether an evacuation sign is installed or not.

An agent represents a group of one to five evacuees. The agent is classified according to the place of residence, the number of constituents, the presence or absence of a physical disability, having a good sense of locality or not, and having evacuation site information or not. An agent whose place of residence is within the map area or has a good sense of locality travels to the evacuation site by the shortest route. Other agents follow evacuation signs or follow nearby agents. In addition, the walking speed of the agent varies depends on the density in the cell and the classification of the agent. Then, the agent supervises evacuation in 
accordance with the above rule on the map in Fig. 1. In this study, the number of evacuees was assumed to be 1000 .

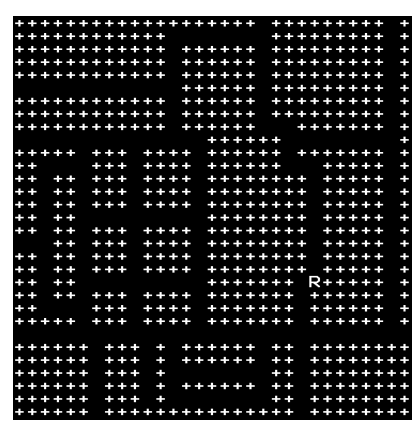

(a) Map1

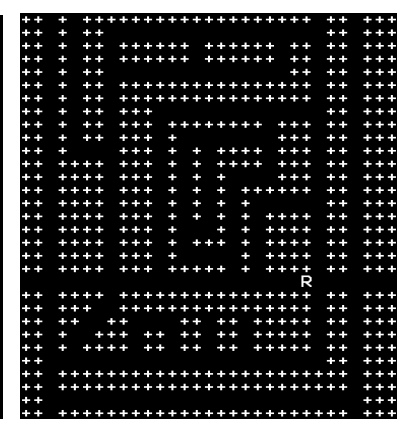

(b) Map2
Figure 1: Evacuation simulation maps in this study: The cell marked by R shows the evacuation-site-adjacent cell.

\section{Proposed Method of Evacuation Sign Arrangement Using Genetic Algorithm}

To optimize the evacuation sign arrangement, we formulate this problem as an optimization problem. Here, the objective function to be minimized consists of the time taken to complete the evacuation and the number of evacuation signs. We define the objective function $f$ as

$$
f=T_{E}+w N_{S}
$$

where $T_{E}$ is the time when the evacuee arrival rate exceeds $90 \%, N_{S}$ is the number of evacuation signs and $w$ is the weight parameter. By minimizing this objective function, it is possible to prevent the installation of ineffective evacuation signs and determine an evacuation sign arrangement that can shorten the evacuation time.

We propose a genetic algorithm. The genetic algorithm is a well-known solver of combinatorial optimization problems. For example, it is used in local facility placement plans [3]. In a genetic algorithm, an initial population of individuals is first generated. Then the selection and the genetic operation, i.e., crossover and mutation, are repeated on the population. Here, we use both elitist selection; ranking selection, and individuals selected by elitist selection are not selected by ranking selection and are not used for crossover.

In our proposed algorithm, the individual is defined by a binary sequence that represents a map in which evacuation signs are arranged. The length of the individual is equal to the number of intersections on the map. Each element of the individual represents whether an evacuation sign is installed or not, i.e., 1 indicates an installed sign and 0 indicates no installed sign. Here we assumed that evacuation signs are installed only at intersections. We show an example of individual coding in Fig. 2.

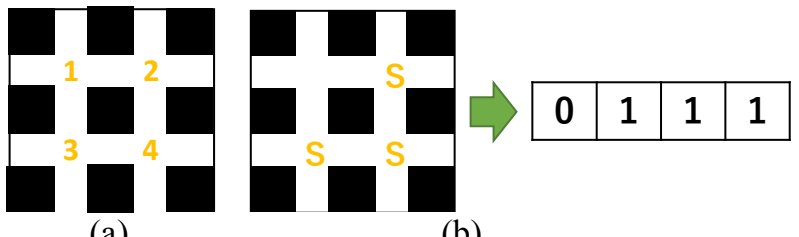

(a)

(b)

Figure 2: Example of an individual: (a) A number is assigned to each intersection, (b) Example of encoding when the signs are installed at intersections marked by $\mathrm{S}$

\section{Simulation Results}

\subsection{Convergence characteristics}

First, to investigate the convergence characteristics of our proposed method, several simulations were carried out using Map1 shown in Fig. 1(a). Figure. 3 shows the convergence characteristics of the objective function. Here, the maximum number of generations was set to 1000 , and the weight parameter $w$ was set to $0,5,10,50$, or 100 .

From these results, it was confirmed that the objective function was minimized regardless of the value of the weight parameter. Also, it was found that a maximum of about 200 generations was sufficient.

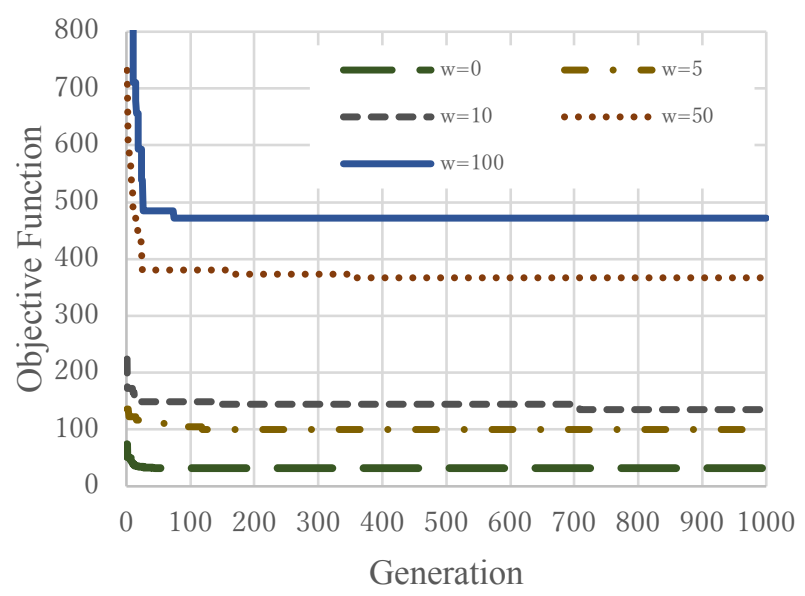

Figure 3: Convergence characteristics of objective function with weight parameters $w$ of $0,5,10,50$, and 100

\subsection{Optimal evacuation sign arrangement}

Our proposed method is tested by numerical experiments. First, to investigate the characteristics of weight parameter $w$, the numerical experiment was repeated for weight parameter $w$ values from 0 to 100 in Eq. (1). Here, the 
numerical experiments were performed using Map1 in Fig. 1(a). Figure 4 shows the dependences of the number of signs on the weight parameter $w$. The number of signs is calculated by counting the signs in the evacuation-sign-arranged map obtained by our proposed method.

As shown in Fig. 4, the number of evacuation signs $N_{S}$ monotonically decreases as the weight parameter $w$ increases. This indicates that suppression of the number of evacuation signs becomes strong as the weight parameter becomes larger.

On the other hand, from these experimental results, we show the evacuation time $T_{E}$ as a function of the number of evacuation signs $N_{S}$ in Fig. 5(a). As shown in Fig. 5(a), as the number of evacuation signs increases, the evacuation time decreases monotonically and eventually saturates. From the results shown in Figs. 4 and 5(a), we can confirm the effect of suppressing the installation of ineffective evacuation signs by adjusting weight parameter $w$.

From the results shown in Fig. 5(a), it is easy to determine a reasonable number of evacuation signs because of its monotonically decreasing and saturation characteristics. Because each solution obtained by a single trial of our method is the optimal evacuation sign arrangement for each $w$, we can choose an appropriate solution from the results in Fig. 5(a) considering the cost-effectiveness. For example, from Fig. 5(a), we find that the effect of increasing the number of evacuation signs on evacuation time is saturated at around 10 or 11 evacuation signs. Thus, a solution in this range should be a good candidate for an optimal solution.

Figures 6(a)-(f) show examples of solution candidates of the evacuation sign arrangement obtained by our method. We can make a final decision appropriately by considering the evacuation time $T_{E}$ and the number of evacuation signs NS.

Furthermore, we tested our proposed method using another map, i.e., Map2 in Fig. 1(b). The evacuation time $T_{E}$ as a function of the number of evacuation signs $N_{S}$ is shown in Fig. 5(b). As with the experiment using Map1, the evacuation time $T_{E}$ monotonically decreases and eventually saturates as the number of evacuation signs increases. We also find that the solutions that recommended around 8 or 9 evacuation signs should be good candidates. Figures 7(a)-(f) show examples of solution candidates of the evacuation sign arrangement obtained by our method.

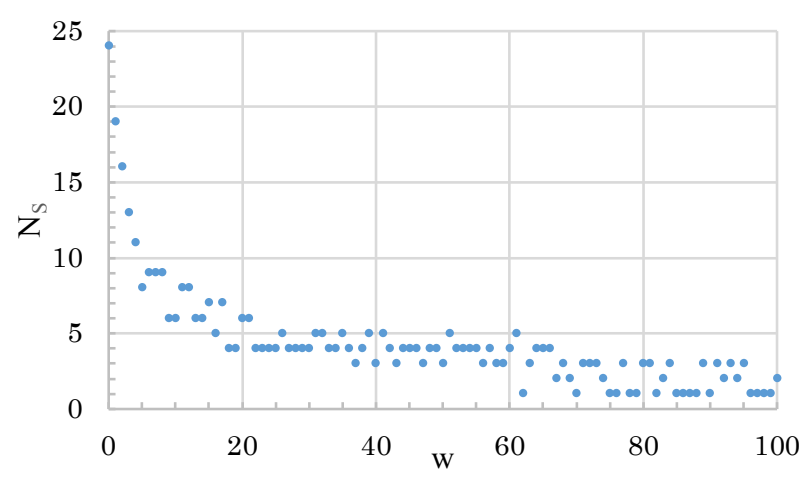

Figure 4: Number of signs $N_{S}$ as a function of weight parameter $w$ obtained using the results of the experiments using Map1

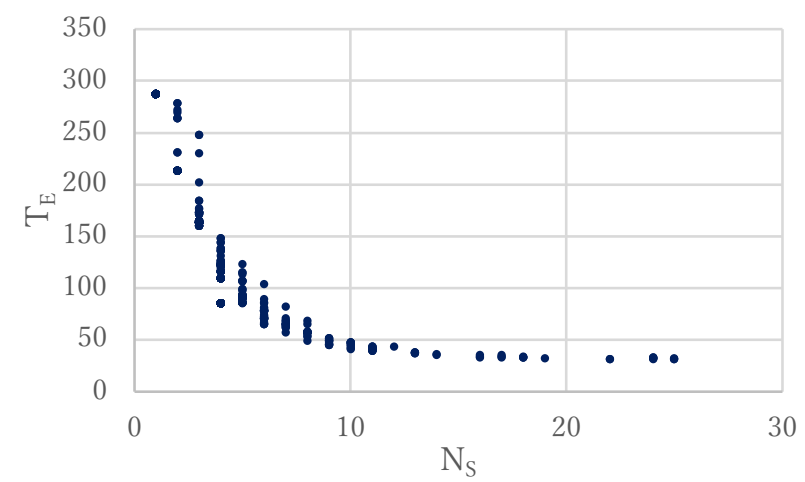

(a) Results obtained from Map1

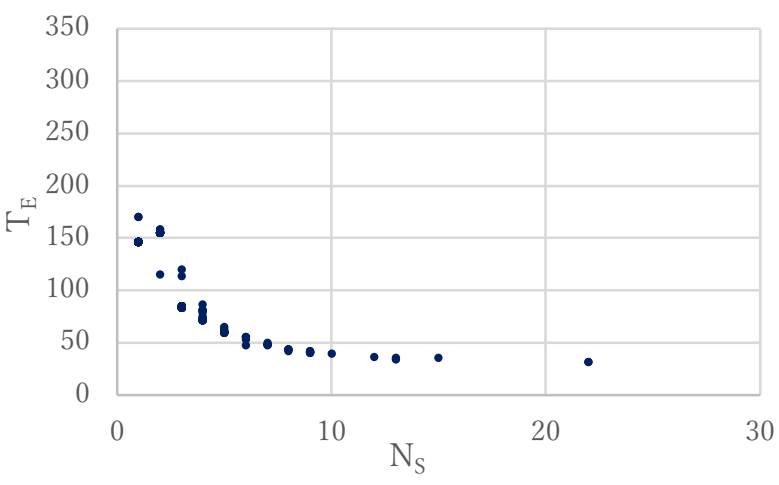

(b) Results obtained from Map2

Figure 5: Evacuation time $T_{E}$ as a function of the number of evacuation signs $N_{S}$ 


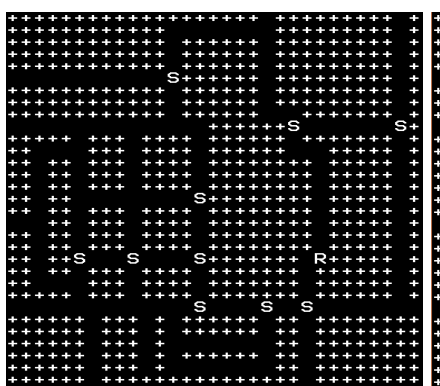

(a) $\mathrm{N}_{\mathrm{S}}=10, \mathrm{~T}_{\mathrm{E}}=41$

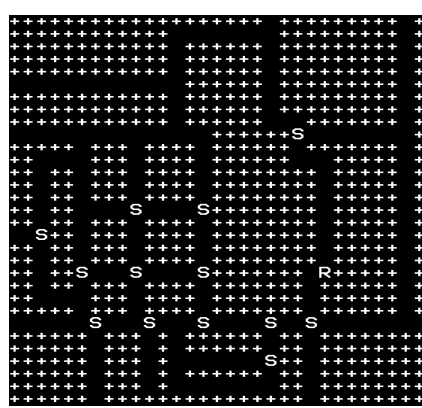

(c) $\mathrm{N}_{\mathrm{S}}=13, \mathrm{~T}_{\mathrm{E}}=37$

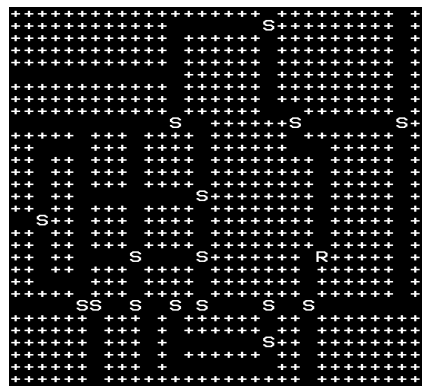

(e) $\mathrm{N}_{\mathrm{S}}=16, \mathrm{~T}_{\mathrm{E}}=33$

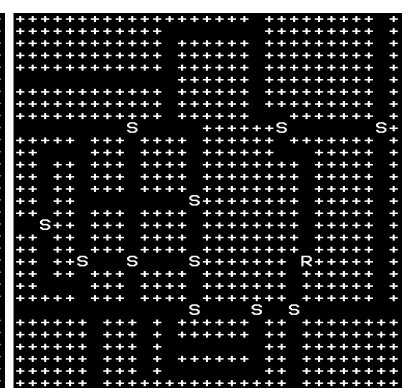

(b) $\mathrm{N}_{\mathrm{S}}=11, \mathrm{~T}_{\mathrm{E}}=39$

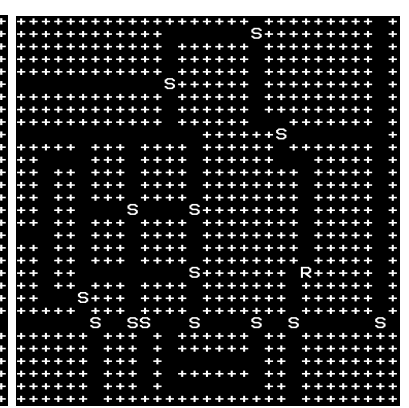

(d) $\mathrm{N}_{\mathrm{S}}=14, \mathrm{~T}_{\mathrm{E}}=35$

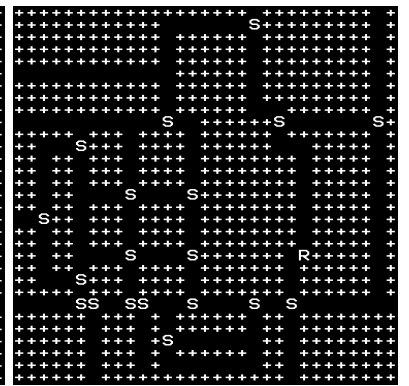

(f) $\mathrm{N}_{\mathrm{S}}=19, \mathrm{~T}_{\mathrm{E}}=32$

Figure 6: Examples of evacuation sign arrangements on Map1 in Fig. 1(a), obtained by proposed method

\section{Conclusions}

In this study, we proposed a method for selecting the installation spots of evacuation signs in a map of a local area without any human experience or knowledge of that area. The proposed method is based on a genetic algorithm, and a multi-agent simulation is used to calculate the objective function. The proposed method can prevent the installation of ineffective evacuation signs by selecting a suitable weight parameter in the objective function.

By numerical simulations, the convergence characteristics and candidate evacuation sign arrangements are shown. The results show that our proposed algorithm can provide several good candidates of the evacuation sign arrangement.
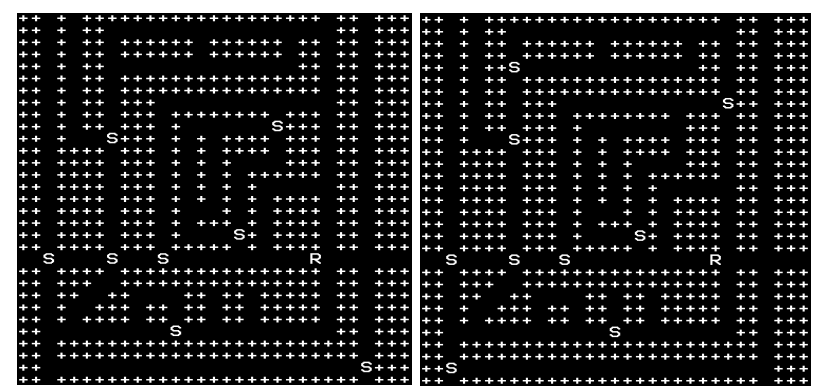

(a) $\mathrm{N}_{\mathrm{S}}=8, \mathrm{~T}_{\mathrm{E}}=42$

(b) $\mathrm{N}_{\mathrm{S}}=9, \mathrm{~T}_{\mathrm{E}}=40$
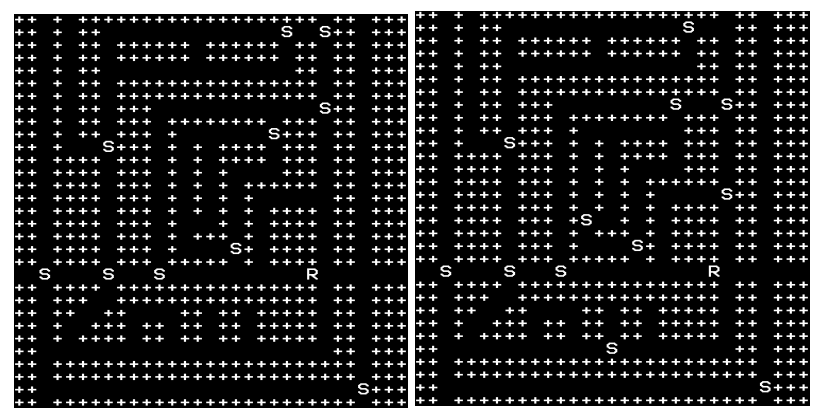

(c) $\mathrm{N}_{\mathrm{S}}=10, \mathrm{~T}_{\mathrm{E}}=39$

(d) $\mathrm{N}_{\mathrm{S}}=12, \mathrm{~T}_{\mathrm{E}}=36$

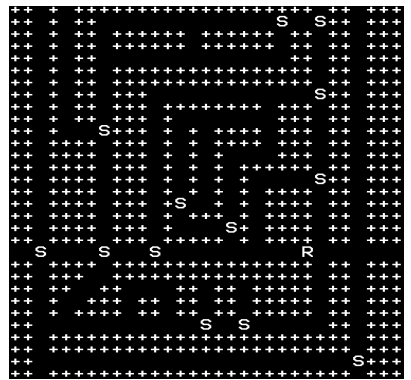

(e) $\mathrm{N}_{\mathrm{S}}=13, \mathrm{~T}_{\mathrm{E}}=34$

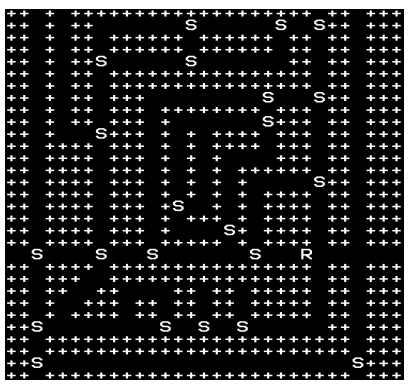

(f) $\mathrm{N}_{\mathrm{S}}=22, \mathrm{~T}_{\mathrm{E}}=31$

Figure 7: Examples of evacuation sign arrangements on Map2 in Fig. 1(b), obtained by proposed method

\section{References}

[1] Y. Muraki and H. Kanoh: Multiagent model for widearea disaster-evacuation simulations with local factors considered, Transactions of the Japanese Society for Artificial Intelligence, Vol. 22, No. 4, pp. 416-424, 2007.

[2] K. Abe and H. Kurokawa: The simulation model of evacuation behavior considering psychological factors of victims, Proc. of NCSP'17, pp. 161-164, 2017.

[3] Y. Aoki and N. Muraoka: A method of the facility location by genetic algorithm, Journal of Architecture, Planning and Environmental Engineering, Vol. 61, No.484, pp. 129-135, 1996. 